\title{
JORDAN ALGEBRAS AND THEIR APPLICATIONS
}

\section{BY KEVIN MCCRIMMON}

In this article I want to sketch for nonexperts what Jordan algebras are why people might want to study such strange objects. I start with the assumption that the reader knows little or nothing about Jordan algebras, but has at least some respect for the terms Lie algebra, Lie group, Riemannian symmetric space, and bounded symmetric domain.

I. Jordan algebras in antiquity (1933-1966). I am unable to prove Jordan algebras were known to Archimedes, or that a complete theory has been found in the unpublished papers of Gauss. Their first appearance in recorded history seems to be in the early 1930's when the theory bursts forth full-grown from the mind, not of Zeus, but of Pascual Jordan, John von Neumann, and Eugene Wigner in their 1934 paper, On an algebraic generalization of the quantum mechanical formalism [14].

In the usual interpretation of quantum mechanics, the observables are Hermitian matrices (or Hermitian operators on Hilbert space) $x^{*}=x$, where the adjoint $x^{*}$ is the conjugate transpose $\bar{x}^{t}$. The basic algebraic operations on such observables $x$ are the matrix operations:

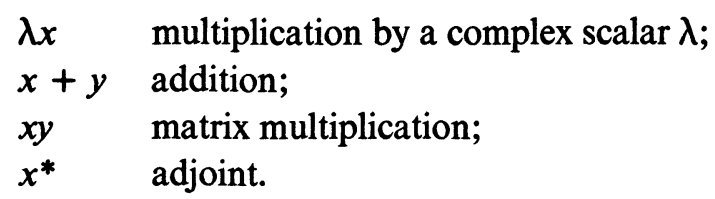

This formulation is open to the objection that the operations are not intrinsic to the physically significant part of the system: the scalar multiple $\lambda x$ is not again Hermitian unless the scalar is real, and the product $x y$ is not again Hermitian unless $x$ and $y$ commute (or, as the physicists say, $x y$ is not observable unless $x$ and $y$ are simultaneously observable). It was philosophically unsatisfactory to derive the algebraic structure from an unobservable operation $x y$, and, in addition, the matrix interpretation seemed insufficient when one attempted to apply quantum mechanics to relativistic and nuclear phenomena.

The program proposed by Jordan was to study the algebraic properties of Hermitian matrices without reference to the underlying (unobservable) matrix algebra. The strategy was:

(1) to formulate formal properties which seemed essential and physically significant;

(2) to consider abstract systems with these same formal properties taken as

An invited address delivered at the 743rd meeting of the Society, April 1, 1977 in Huntsville, Alabama; received by the editors September 6, 1977.

AMS (MOS) subject classifications (1970). Primary 17Cxx, 17B25, 17B60, 32M15. 
axioms, and to determine what other systems satisfy these axioms.

The hope was to find a system which wasn't a system of Hermitian matrices, but acted like one.

The observable operations on Hermitian matrices or operators were

$\begin{array}{ll}\alpha x & \text { multiplication by a real scalar } \alpha ; \\ x+y & \text { addition; } \\ x \circ y=x y+y x & \text { symmetric product; } \\ x y x & \text { quadratic product; } \\ x^{n} & \text { powers }(n=0,1,2, \ldots) .\end{array}$

Note that if $x^{*}=x, y^{*}=y$ are Hermitian, so are $x y+y x, x y x$, and $x^{n}$.

After a little empirical experimentation, it seemed that two basic identities of degrees 2 and 4 implied all the others. Jordan took as his axioms the existence of a bilinear product $x \circ y$ on a real vector space satisfying the identities:

$$
\begin{aligned}
& x \circ y=y \circ x \quad \text { (commutative law), } \\
& \left(x^{2} \circ y\right) \circ x=x^{2} \circ(y \circ x) \quad \text { (Jordan identity). }
\end{aligned}
$$

Such systems are now called (linear) Jordan algebras. In the fundamental 1934 paper the authors showed that all such finite-dimensional "formally real" systems were direct sums of simple systems, where there were 5 simple building blocks:

$$
H_{n}(\mathbf{R}), \quad H_{n}(\mathbf{C}), \quad H_{n}(\mathbf{H}), \quad H_{3}(\mathbf{O}), \quad J(Q)
$$

where $H_{n}(A)$ denotes Hermitian $n \times n$ matrices with entries in $A$ (the reals $\mathbf{R}$, complexes $\mathbf{C}$, quaternions $\mathbf{H}$, octonions or Cayley numbers $\mathbf{0}$ ), and multiplication in $J(Q)$ is given in terms of a real-valued quadratic form $Q$ by $x \circ y=Q(x, y) 1$.

Call a linear Jordan algebra special if it can be imbedded in an associative algebra so that $x \circ y=x y+y x$. Thus in a special Jordan algebra the algebraic structure $x \circ y$ is derived from an ambient associative structure $x y$. The first 3 building blocks in (1.2) clearly lie in associative matrix algebras, and the fifth is imbedded in the Clifford algebra of $Q$. The fourth example $\mathrm{H}_{3}(\mathrm{O})$ does not seem to be special, since its coordinates come from the nonassociative algebra $O$ of Cayley numbers. In 1934 A. A. Albert [1] showed that $\mathrm{H}_{3}(\mathbf{O})$ actually is not special, thus is exceptional: it cannot be imbedded in any associative algebra whatsoever. ${ }^{1}$

\footnotetext{
${ }^{1}$ It is not even the homomorphic image of a special algebra. P. M. Cohn [4] was the first to show that a special Jordan algebra $J \subset A$ could have a homomorphic image $J / I$ which was not special (taking a Jordan ideal $I$ which was not the restriction $B \cap J$ of an associative ideal $B$ of $A$ ). Thus the special Jordan algebras do not form a variety defined by identities. However, by a general theorem of Birkhoff, the special algebras together with their homomorphic images do form a variety, and are therefore characterized by the Jordan identity plus some "special" or "s-identities" valid in all special algebras (therefore their homomorphic images as well), but not in all Jordan algebras. THESE IDENTITIES HAVE NEVER BEEN FOUND. To date only two s-identities are known, the Glennie identities $G_{8}$ and $G_{9}$ of degrees 8 and 9 in 3 variables $x, y, z$. The modern proof of exceptionality of $H_{3}(0)$ is to check that Glennie's identity holds in all special Jordan algebras but fails in $H_{3}(0)$ for judicious choice of $x, y, z$. No one knows whether there are infinitely many essentially different $s$-identities (i.e. if the ideal of all $s$-identities in the free Jordan algebra is finitely generated).
} 
Thus after isolating the basic algebraic properties of Hermitian matrices and investigating these properties axiomatically, what resulted were special algebras plus one lone exceptional algebra $\mathrm{H}_{3}(\mathrm{O})$ (of degree 3 and dimension 27). As the authors said, "Physical considerations demand an algebra essentially more general than matrix algebras.... The one Jordan algebra which is not a matrix algebra is too narrow for the generalization needed." Having met with failure in the search for a new algebra, the authors held out a slight hope: "One thinks of omitting the condition of finiteness, since the algebra appearing in quantum mechanics is infinite. It may well happen that new types of algebra will arise with the removal of restrictions... in ordinary quantum mechanics many important features first appear in infinite algebras" [12, p. 30].

In 1936 von Neumann made an initial study [28] of some infinite dimensional algebras (Jordan " $W^{*}$-algebras"); as axiom he took power associativity $x^{n} x^{m}=x^{n+m}($ or $f(g(x))=(f \circ g)(x)$ for all polynomials $f, g)$, and showed this was equivalent to the Jordan identity in the formally real case. This cast Jordan algebras in a more natural light, deriving everything from the operations of raising $x$ to powers $x^{n}$ (the product $x \circ y$ is obtained by linearizing the square $x^{2}$ ).

Just recently, in 1975, Eric Alfsen, Frederic Schultz, and Erling Störmer in an important work [2] derived a Gelfand-Naimark Theorem for Jordan $C^{*}$-algebras (Jordan algebra structures on Banach spaces satisfying $\|a \circ b\|<$ $\|a\|\|b\|,\left\|a^{2}\right\|=\|a\|^{2}$, and a formal reality condition $\left.\left\|a^{2}\right\|<\left\|a^{2}+b^{2}\right\|\right)$ showing the basic building blocks for such algebras are either special (contained in ordinary $C^{*}$-algebras) or 27 -dimensional exceptional. Their idea, following the associative case, was to imbed the Jordan $C^{*}$-algebra in its Arens double dual, which was a Jordan $W^{*}$-algebra with lots of idempotents. Speciality comes easily in the presence of idempotents; for example, any simple Jordan algebra with 4 orthogonal idempotents is special [24].

Thus any new exceptional Jordan algebra cannot admit many idempotents-it must be poor in projections. TO THIS DATE NO ONE HAS EVER FOUND A SIMPLE EXCEPTIONAL JORDAN ALGEBRA WHICH IS NOT 27-DIMENSIONAL OVER ITS CENTER. ${ }^{2}$

At this point the physicists largely abandoned Jordan algebras, and the algebraists took over: A. A. Albert, Nathan Jacobson, and others developed a complete theory of finite-dimensional Jordan algebras over arbitrary fields of characteristic $\neq 2$.

It would be most unfair to picture lions finishing a kill and leaving the remains to the jackals. For one thing, physicists have not finished feeding on the carcass. Feza Gürsey [7] in 1974 proposed a role for the 27-dimensional

\footnotetext{
${ }^{2}$ A good place to look is the free algebra: the free Jordan algebras on 3 or more generators are exceptional, and if they behave like free associative algebras [5] they are domains imbeddable in Jordan division rings, and such division rings would be both simple and exceptional and infinite-dimensional over their center. [On the other hand, the free Jordan algebras may share the pathologies of free alternative rings, which are at the opposite extreme from domains: they contain nilpotent elements. It is not known which way free Jordan rings will go, whether they are domains or whether they contain zero divisors, perhaps even nilpotent elements-in fact it is not even known if a free Jordan algebra can have trivial elements $z$ with $U_{z} J=0$.]
} 
exceptional Jordan algebra in elementary particle physics, to explain charmed and colored quarks and confinement of quarks. Also, physicists seeking supersymmetry looked for algebraic systems encompassing both bosons and fermions; such systems require a Lie-like part and a Jordan-like part. This led to the investigation of Lie and Jordan superalgebras, whose classification (in characteristic 0) was just completed in the summer of 1976 by Irving Kaplansky [16] and Victor Kac [15].

For another thing, these Jordan algebras (especially the 27-dimensional exceptional algebra) which arose in an unsuccessful attempt to find a new algebra suitable for quantum mechanics turned out to have unforseen applications to Lie groups and algebras, geometry, and analysis.

II. Jordan algebras in modern times (1966-present). The modern theory of Jordan algebras may be said to date from the appearance in 1966 of $\mathrm{N}$. Jacobson's paper, Structure theory for a class of Jordan algebras [12]. Fastening on the notion of quadratic (or inner) ideal which appeared incidentally in the work of D. M. Topping [33] on Jordan algebras of selfadjoint operators, Jacobson showed how this concept played the role of one-sided ideals and could be used to develop a ring-theoretic treatment of Jordan algebras. Here the basic operation is $U_{x} y=x y x$ instead of $x \circ y=x y+y x$, and the axioms (due to K. McCrimmon [23], [11]) are

$$
\begin{array}{ll}
\text { (Q1) } & U_{1}=\mathrm{Id}, \\
\text { (Q2) } & U_{x} V_{y, x}=V_{x, y} U_{x}, \\
\text { (Q3) } & U_{U(x) y}=U_{x} U_{y} U_{x},
\end{array}
$$

where $V_{x y}(z)=\{x y z\}=\left(U_{x+z}-U_{x}-U_{z}\right) y$. Since the product $U_{x} y$ is quadratic in the variable $x$, the resulting systems are called quadratic Jordan algebras. For example, any associative algebra $A$ determines a quadratic Jordan algebra $A^{+}$with product $U_{x} y=x y x$ :

$$
\begin{gathered}
1 a 1=a, \quad x(y x a+a x y) x=x y(x a x)+(x a x) y x, \\
(x y x) a(x y x)=x(y(x a x) y) x .
\end{gathered}
$$

Any subspace of $A$ containing 1 and closed under $x y x$ will form a Jordan algebra. For example, if $A$ has an involution*, the set $H\left(A,{ }^{*}\right)$ of symmetric elements $x^{*}=x$ forms an archetypal Jordan algebra.

The theories of linear and quadratic Jordan algebra are categorically equivalent when there is a scalar $\frac{1}{2}$. Whereas the linear theory breaks down in characteristic 2 or whenever there is no $\frac{1}{2}$, the quadratic theory works in all characteristics and, more importantly (since most people are quite willing to toss characteristic 2 to the lions), over an arbitrary ring of scalars. In particular, it is possible to carry out arithmetic investigations.

The quadratic approach via the $U$-operators reveals the essential algebraic properties of Jordan algebras much more clearly than the linear approach via the $V$-operators $\left(V_{x} y=x \circ y\right)$ :

(2.2) $x$ is invertible iff the operator $U_{x}$ is invertible, in which case $U_{x^{-1}}=$ $U_{x}^{-1}$, but $V_{x}$ need not be invertible, nor $V_{x^{-1}}=V_{x}^{-1}$-for example, in the division algebra of real quaternions we have $V_{i} j=i \circ j=0$. 
(2.3) If $B, C$ are ideals in $J$ then $U_{B} C$ is an ideal, but $B \circ C$ need not be. Thus the correct notion of solvability depends on the quadratic composition.

(2.4) The generic norm (an analogue of the reduced norm of associative theory, generalizing the determinant) admits composition with $U$ but not with $V: N\left(U_{x} a\right)=N(x) N(y) N(x)$, but $N(x \circ y)=$ nothing (e.g. $\operatorname{det} x y x=$ $(\operatorname{det} x)(\operatorname{det} y)(\operatorname{det} x)$, but $\operatorname{det}(x y+y x)=$ ?).

(2.5) A left ideal in the associative ring $A$ is a subspace $B$ closed under left multiplication by the ambient ring $A$. The product $U_{x} y=x y x$ in a Jordan ring $J$ has no left or right, only an outside and an inside. An inner ideal is a subspace $B$ closed under inner multiplication by $J, U_{B} J \subset B$. Thus the concept of an inner ideal arises naturally in the quadratic theory and plays the role of 1 -sided ideals, whereas the commutative product $x \circ y$ admits no 1-sided ideals.

For these quadratic rings, there is an analogue of the Artin-Wedderburn structure theory for associative rings with dcc on left ideals (compare (1.2)).

(2.6) STRUCTURe TheOREM [11], [23]. A nondegenerate quadratic Jordan ring with descending chain condition on inner ideals is a direct sum of a finite number of simple ideals. These ideals are (up to isotopy) essentially of the form:

(0) Jordan division rings,

(1) $H_{n}(\Delta)$ for some associative division ring $\Delta$ with involution,

(2) $H_{n}\left(\Delta \oplus \Delta^{\mathrm{op}}\right)=M_{n}(\Delta)^{+}$for some associative division ring $\Delta$,

(3) $H_{n}(Q)$ for some split quaternion algebra $Q$,

(4) $\mathrm{H}_{3}(\mathbf{O})$ for some octonion (Cayley) algebra $\mathrm{O}$,

(5) a Jordan algebra $J(Q, c)$ determined by a quadratic form $Q$.

Here (1), (2), (3) are precisely all $H\left(A,{ }^{*}\right)$ for ${ }^{*}$-simple associative artinian rings with involution.

We must stress that quadratic Jordan algebras should be thought of as axiomatic characterizations of the product $x y x$ in the space of Hermitian matrices (or, more generally, $H\left(A,{ }^{*}\right)$ ). IT IS NOT A QUESTION OF A BILINEAR PRODUCT $x \circ y$ WHICH IS NONASSOCIATIVE, BUT RATHER A QUADRATIC PRODUCT $U_{x} y$ WHICH IS AS ASSOCIATIVE AS SUCH A PRODUCT CAN BE (i.e. (2.1)). It is misleading to think of Jordan algebras as based on the product $x y+y x$ in the way that Lie algebras are based on the product $x y-y x$; Jordan algebras are closed under the additional product $x y x$, for which no Lie analogue exists, and it is this extra product which makes Jordan theory work smoothly in all characteristics (whereas Lie theory works well only in characteristic 0 ).

III. Isotopes and structure groups. Next we consider two more aspects of Jordan theory which stand out more clearly in the quadratic approach, and are important in applications.

Given an invertible element $u$ in an associative algebra $A$, we can form a new algebra, the $u$-isotope $A^{[u]}$, having the same linear structure but new twisted product

$$
x_{\cdot u} y=x u^{-1} y .
$$

If you stick a $u^{-1}$ in the middle of an associative product it keeps right on 
associating, so we have a new associative algebra. The unit element and inverse are easily computed in this new structure:

$$
1^{[u]}=u, \quad x^{-1[u]}=u x^{-1} u,
$$

since $u_{\cdot u} x=u u^{-1} x=x=x u^{-1} u=x \cdot{ }_{u} u$ shows $u$ is a right and left unit, and when $x$ is invertible in the original algebra we have $u x^{-1} u_{\cdot u} x=$ $u x^{-1} u u^{-1} x=u=1^{[u]}$ and, similarly, $x_{\cdot u} u x^{-1} u=1^{[u]}$.

The isotope $A^{[u]}$ is really just the algebra $A$ in disguise, for $x \rightarrow x u$ is an isomorphism $A \rightarrow A^{[u]}$. Thus in the associative case, isotopy, which basically is just changing the unit element, does not lead to new structures. Nevertheless, it can still be useful. For example, the concept of isotopy plus a little third grade arithmetic allows us to establish the Hua identity,

$$
\left(a+a b^{-1} a\right)^{-1}+(a+b)^{-1}=a^{-1},
$$

which arose in projective geometry [9] and is delicate to verify directly. Now the weak Hua identity,

$$
\left(1+b^{-1}\right)^{-1}+(1+b)^{-1}=1,
$$

where $a=1$ is verifiable by third graders:

$\frac{1}{1+b^{-1}}+\frac{1}{1+b}=\frac{b}{b} \frac{1}{1+b^{-1}}+\frac{1}{1+b}=\frac{b}{b+1}+\frac{1}{1+b}=\frac{1+b}{1+b}=1$.

The case for general $a$ is not so elementary, as noncommutative algebra is not usually taught in the third grade. Fortunately, every Jordan algebraist is equipped with a magic wand which can convert any element to 1 upon uttering the magic word "isotope". That is, we introduce an isotopic algebra $A^{[a]}$ in which $a$ becomes the rightful unit. By our third grade methods we know the weak Hua identity (3.4) holds in $A^{[a]}$, and in view of the formulas (3.2) this means

$$
a\left(a+a b^{-1} a\right)^{-1} a+a(a+b)^{-1} a=a=a a^{-1} a .
$$

Cancelling $a$ 's fore and aft, we get the general Hua identity (3.3). Thus the general Hua identity (3.3) is just the validity of the weak Hua identity (3.4) in all isotopes.

In Jordan algebras we have an entirely analogous notion of isotopy. In an associative isotope $A^{[u]}$ the Jordan product is given by $x_{\cdot_{u}} y_{\cdot_{u}} x=$ $x u^{-1} y u^{-1} x$; in general, we define the $u$-isotope $J^{[u]}$ to have quadratic product.

$$
U_{x}^{[u]} y=U_{x} U_{u}^{-1} y
$$

This new Jordan structure has unit element $1^{[u]}=u$, so once more isotopy amounts to shifting the unit. However, in the Jordan case this can produce an essentially different algebra: $J^{[u]}$ need not be isomorphic to $J$. For example, the algebra $J=H_{n}(\mathbf{R})$ of real $n \times n$ symmetric matrices is "formally real", in particular, has no nonzero nilpotent elements (each symmetric $x$ being diagonalizable, where a power of a diagonal matrix vanishes only when each diagonal entry vanishes), whereas the isotope $J^{[u]}$ for 


$$
u=\left(\begin{array}{lllll}
0 & 1 & & & \\
1 & 0 & & & \\
& & 1 & \ddots & \\
& & & & 1
\end{array}\right)
$$

has nontrivial nilpotent element

$$
e=\left[\begin{array}{cccc}
1 & 0 & & \\
0 & 0 & & \\
& & 0 & \\
& & & 0
\end{array}\right], \quad e^{2[u]}=e u^{-1} e=0 .
$$

The concept of two Jordan algebras being isotopic (each being isomorphic to an isotope of the other) is thus more general than being isomorphic. Many important properties of Jordan algebras are unaffected by isotopy, and the attempt to describe a Jordan algebra plus all of its isotopes in a single algebraic structure has led to the concept of a Jordan pair (which we will meet again).

Isotopy is intimately connected with our second concept, the structure group $\operatorname{Str}(J)$ of a Jordan algebra. This was introduced by M. Koecher [17] as the set of all invertible linear transformations $T$ on $J$ for which there is an invertible $T^{*}\left(=T^{-1} U_{T 1}\right)$ such that

$$
U_{T x}=T U_{x} T^{*} \quad \text { for all } x \in J \quad(T \in \operatorname{Str}(J)) \text {. }
$$

By its definition, this is an algebraic group. Jacobson had studied it earlier as the set of norm similarities, since for a semisimple finite-dimensional algebra over a field, (3.6) is equivalent to

$$
N(T x)=\tau N(x) \text { for all } x \in J
$$

( $N$ the generic norm of $J, \tau=\tau(T)$ a scalar depending only on $T$ ).

A more illuminating way to regard the structure group is as the group of autotopies of $J$ (isomorphisms of $J$ with its isotopes, $J \stackrel{T}{\rightarrow} J^{[T 1]}$, or, equivalently, of one isotope with another, $J^{[u]} \stackrel{T}{\rightarrow} J^{[T u]}$ ).

The automorphism group $\operatorname{Aut}(J)$ is just the set of autotopies fixing the unit element 1. More generally, two isotopes $J^{[u]}, J^{[v]}$ are isomorphic iff there exists an element of the structure group sending $u$ into $v$, so the isomorphism classes of isotopes are in 1-1 correspondence with the orbits of the structure group (acting on the invertible elements of $J$ ).

The Hua identity is valid for Jordan algebras,

$$
\left(a+U(a) b^{-1}\right)^{-1}+(a+b)^{-1}=a^{-1},
$$

and again follows from the weak Hua identity via isotopy. This identity was used by T. A. Springer (following up earlier ideas of Koecher) in his 1973 book, Jordan algebras and algebraic groups [29], to base the entire theory of finite-dimensional Jordan algebras upon the inverse. His axioms for a $J$ structure were:

(i) the existence of a birational inversion $j$ (involutary of degree -1 );

(ii) a unit $e$ and the weak Hua identity 


$$
j(e+j x)+j(e+x)=e ;
$$

(iii) density of the orbit of $e$ under the structure group (defined as the invertible $T$ with $j(T x)=T^{*-1} j(x)$ ).

There were snags in his theory in characteristic 2; the axioms he should have taken were those for an $\boldsymbol{H}$-structure [25]:

(i) existence of an inversion $j$;

(ii) existence of a unit $e: j(e)=e$ and $U_{e}=\mathrm{Id}$ (for $U_{x}$ defined as the negative inverse of the differential of $j, U_{x}=\left(\left.d j\right|_{x}\right)^{-1}$;

(iii) the validity of the weak Hua identity in all isotopes:

$$
j^{[u]}\left(e^{[u]}+j^{[u]}(x)\right)+j^{[u]}\left(e^{[u]}+x\right)=e^{[u]}
$$

for $j^{[u]}=U_{u} \circ j, e^{[u]}=u$, i.e. the general Hua identity $j\left(u+U_{u} j(x)\right)+j(u+$ $x)=j(u)$.

This axiomatically characterizes the inversion map $j(x)=x^{-1}$ in quadratic Jordan algebras: $\boldsymbol{H}$-structures are categorically equivalent to Jordan algebras.

The Hua identity (3.8) is equivalent to the representability of the inversion by the geometric series, i.e. that

$$
j(e-t x)=\sum_{0}^{\infty} t^{n} x^{n}
$$

be valid (as formal power series) in all isotopes. Thus in terms of their inverses, Jordan algebras are singled out as precisely those algebraic systems whose inverse is given by the geometric series.

It is important that all algebraic information about a Jordan algebra is encoded in the inverse. Springer developed the entire theory of finite-dimensional Jordan algebras in terms of inverses (including classification via the structure group), completely avoiding the nonassociative product $x \circ y$ or the quadratic product $U_{x} y$, making the entire theory more accessible to algebraic group theorists. It must be stressed that this approach is essentially limited to the finite-dimensional case, or other situations where a rich supply of invertible elements exists.

IV. Applications to projective geometry. Now we turn to applications, beginning with geometry. In 1933 Ruth Moufang [27] constructed a projective plane coordinatized by a Cayley division ring. Because the coordinates were not associative, the resulting plane was not Desarguian. However, it did have the Little Desargues Property and the Harmonic Point Property, and in fact any Little Desarguian plane is either Desarguian or one of these Moufang planes. The coordinatization by a Cayley algebra was insufficient for a description of the automorphisms (collineations) of the plane-a "fundamental theorem of geometry" was not obtained.

In 1949 Jordan (and, independently, H. Freudenthal in 1951) coordinatized these planes by the exceptional Jordan algebra $J=H_{3}(0)$. Points and lines were rays $\mathbf{R} z$ determined by the "rank 1" elements of $J$ (those with $U_{z} J=\mathbf{R} z$ of dimension 1; these were just primitive idempotents in suitable isotopes). Such a point $x$ and line $y$ were incident if $t(x, y)=0$ in terms of the generic trace $t$. The elements of this geometry $\pi(J)$ lay in the exceptional algebra $J$, 
and their geometric relations were described in Jordan algebra terms. Moreover, with this description the collineation group could be easily characterized.

4.1. Fundamental Theorem of Projective Geometry for Moufang Planes $\pi(J)$ (Springer [30]; Jacobson [10], 1960; Faulkner [6], 1970). The collineation group of the Moufang plane $\pi(J)$ is the semilinear structure group (the semilinear autotopies, or norm semisimilarities), and the little projective group generated by relations is the linear structure group. Two planes $\pi(J), \pi\left(J^{\prime}\right)$ are isomorphic iff the algebras $J, J^{\prime}$ are isotopic.

Once more the concepts of isotopy and structure group play a crucial role.

V. Applications to Lie algebras and algebraic groups. Jordan methods have proved useful in the study of other algebraic systems, such as associative rings with involution [26], right alternative rings [31] and noncommutative Jordan rings [22], but their most important application in nonassociative algebra is to Lie algebras. Indeed, much of the algebraists' interest in Jordan algebras (especially the exceptional ones) during the 30's and 40's was due to their close connections with the exceptional Lie algebras. Although these Lie algebras could be constructed directly from their Cartan matrices, this was not illuminating; one needed a workable model. Exceptional Jordan algebras provided such a model. We can relate the 5 exceptional types of simple Lie algebras to the Cayley algebra $\mathrm{O}$ and exceptional Jordan algebra $H_{3}(\mathbf{O})$ as follows.

\subsection{Table of Exceptional Lie Algebras [13]}

\begin{tabular}{|c|c|c|c|}
\hline Type & Lie Algebra & Lie (or algebraic) group & $\underline{\text { Dimension }}$ \\
\hline$G_{2}$ & Derivations of $\mathbf{O}$ & Automorphisms of $\mathbf{O}$ & 14 \\
\hline$F_{4}$ & Derivations of $H_{3}(\mathbf{O})$ & Automorphisms of $H_{3}(\mathbf{O})$ & 52 \\
\hline$E_{6}$ & $\begin{array}{l}\text { Reduced structure } \\
\text { algebra } \operatorname{Strl}_{0}(J)=\operatorname{Der} J+V_{J_{0}}\end{array}$ & $\begin{array}{l}\text { Reduced structure group } \\
\operatorname{Str}(J) / \mathbf{R} \text { Id of } H_{3}(\mathbf{O})\end{array}$ & $52+(27-1)=78$ \\
\hline$E_{7}$ & $\begin{array}{l}\text { Superstructure algebra } \\
J \oplus \operatorname{Strl}(J) \oplus \bar{J} \text { of } H_{3}(\mathbf{O})\end{array}$ & Superstructure group of $\mathrm{H}_{3}(\mathbf{O})$ & $27+79+27=133$ \\
\hline$E_{8}$ & $?$ & $?$ & 248 \\
\hline
\end{tabular}

Here $J_{0}$ denotes the elements of $J$ having (generic) trace 0 . To obtain $E_{8}$ as well, one needs a striking general recipe due to Tits in 1966 [32], [13].

\subsection{Freudenthal-Tits Magic SQuare}

Ingredients: a composition algebra $A$, a Jordan algebra $J$ of degree 3 Recipe: $L=\operatorname{Der}(A) \oplus\left(A_{0} \otimes J_{0}\right) \oplus \operatorname{Der}(J)$, with Lie products

$$
\begin{gathered}
{[D, a \otimes x]=D(a) \otimes x, \quad[E, a \otimes x]=a \otimes E(x),} \\
{[a \otimes x, b \otimes y]=\frac{1}{2} t(x, y) D_{a, b}+\left(a^{*} b\right) \otimes\left(x^{*} y\right)-t(a, b) D_{x, y}=u g h !}
\end{gathered}
$$

Resulting type of algebra: 


\begin{tabular}{c|ccccc}
$J$ & $\mathbf{R}$ & $H_{3}(\mathbf{R})$ & $H_{3}(\mathbf{C})$ & $H_{3}(\mathbf{H})$ & $H_{3}(\mathbf{O})$ \\
\hline $\mathbf{R}$ & 0 & $A_{1}$ & $A_{2}$ & $C_{3}$ & $F_{4}$ \\
$\mathbf{C}$ & 0 & $A_{2}$ & $A_{2} \oplus A_{2}$ & $A_{5}$ & $E_{6}$ \\
$\mathbf{H}$ & $A_{1}$ & $C_{3}$ & $A_{5}$ & $A_{6}$ & $E_{7}$ \\
$\mathbf{O}$ & $G_{2}$ & $F_{4}$ & $E_{6}$ & $E_{7}$ & $E_{8}$
\end{tabular}

For example, in the bottom corner when $A=\mathbf{O}, J=H_{3}(\mathbf{O})$, the Lie algebra Der $A \oplus\left(A_{0} \otimes J_{0}\right) \oplus \operatorname{Der} J$ will have dimension $14+(7 \times 26)+52=248$.

Both I. L. Kantor and Koecher discovered methods of "imbedding" a Jordan algebra in a Lie algebra.

\subsection{Tits-Koecher Construction of Superstructure Algebra}

$$
\begin{gathered}
K(J)=J \oplus \operatorname{Strl}(J) \oplus \bar{J}, \\
{[W, x]=W(x), \quad[W, \bar{x}]=\overline{W^{*}(x)}, \quad[x, \bar{y}]=V_{x y} .}
\end{gathered}
$$

This correspondence is functorial, so important information about $J$ can be read off from the Lie algebra $K(J)$ (e.g. $J$ is simple or semisimple iff $K(J)$ is). Isotopic Jordan algebras produced isomorphic Lie algebras, and the structure group of $J$ induces a group of automorphisms of $K(J)$. In view of this Kantor has said "There are no Jordan algebras, there are only Lie algebras".

Besides these methods of building Lie algebras starting from a Jordan algebra, we can start from a suitable Lie algebra and derive a Jordan algebra from it. Acutally, what we get is slightly more general than a Jordan algebra. We will now say a few words about these systems (though the reader should not get bogged down in the identities).

A Jordan triple system is just a Jordan algebra with the unit thrown away. For example, the rectangular $p \times q$ matrices $M_{p, q}(\mathbf{R})$ form a triple system under the product $x y^{t} x$. Axiomatically:

$$
\begin{aligned}
& V_{x, y} U_{x}=U_{x} V_{y, x}, \\
& V_{U(x) y, y}=V_{x, U(y) x}, \\
& U_{U(x) y}=U_{x} U_{y} U_{x} .
\end{aligned}
$$

In such systems there is no bilinear product $x \circ y$ or square $x^{2}$. The term "triple system" is due to the fact that when one can divide by 2 and 3, the system is completely described by the trilinear product $\{x y z\}=V_{x y} z$, and the triple system axioms can be simplified to

$$
\left[V_{x, y}, V_{z, w}\right]=V_{V(x, y) z, w}-V_{z, V(y, x) w}
$$

A Jordan pair $V=\left(V_{+}, V_{-}\right)$is just a pair of spaces acting on each other like Jordan triple systems. For example, we can take $V_{+}$as $p \times q$ matrices $M_{p, q}(R)$ and $V_{-}$as $q \times p$ matrices $M_{q, p}(R)$ under the action $U_{x} y=x y x$. Axiomatically, we have two quadratic products $U_{x_{\varepsilon}} y_{-\varepsilon} \in V_{\varepsilon}(\varepsilon= \pm 1)$ satisfying 


$$
\begin{gathered}
V_{x_{\varepsilon}, y_{-e}} U_{x_{\varepsilon}}=U_{x_{\varepsilon}} V_{y_{-e}, x_{e}}, \\
V_{U\left(x_{e}\right) y_{-e} y_{-e}}=V_{x_{\varepsilon}, U\left(y_{-e}\right) x_{e}}, \\
U_{U\left(x_{e}\right) y_{-e}}=U_{x_{\varepsilon}} U_{y_{-e}} U_{x_{e}} .
\end{gathered}
$$

One way to get a Jordan pair $V$ is to pair a Jordan triple system or Jordan algebra $J$ with itself, $V_{+}=V_{-}=J$ and $U_{x_{2}} y_{-\varepsilon}=U_{x} y$. In the first case the resulting pair has an involution $V_{-} \rightarrow V_{+}$, and in the second the pair contains invertible elements $\left(U_{1_{+}}\right.$is a bijection $\left.V_{-} \rightarrow V_{+}\right)$. Conversely, every Jordan pair with involution or invertible elements results by doubling a Jordan triple system or algebra:

Jordan triple systems $=$ Jordan pairs with involution, Jordan algebras up to isotopy $=$ Jordan pairs with invertible elements.

Any Lie algebra with a 3-grading $L=L_{1} \oplus L_{0} \oplus L_{-1}\left(\left[L_{i}, L_{j}\right] \subset L_{i+j}\right)$ is obtained by generalizing the Tits-Koecher Construction 5.3.

(5.7) THEOREM. There is a 1-1 correspondence between 3-graded Lie algebras $L=L_{1} \oplus L_{0} \oplus L_{-1}$ (having no ideal in $L_{0}$ ) and Jordan pairs $V=\left(V_{+} V_{-}\right)$ (plus choice of subalgebra $L_{0}$ in between Der $V$ and Inder $V$ ). The Jordan pair $V(L)$ associated with $L$ is given by $V_{+}=L_{1}, V_{-}=L_{-1}$, with product $\left\{x_{\varepsilon} y_{-\varepsilon} z_{\varepsilon}\right\}=\left[\left[x_{\varepsilon} y_{-\varepsilon}\right], z_{\varepsilon}\right]$. The Lie algebra $L(V)$ associated with $V$ is $V_{+} \oplus L_{0}$ $\oplus V_{-}$with Lie products $\left[x_{+}, y_{-}\right]=V_{x_{+} y_{-}}$and $\left[D_{0}, x_{+}\right]=D_{0}(x)_{+},\left[D_{0}, y_{-}\right]$ $=D_{0}^{*}(y)_{-}$.

The Jordan pair $V(L)$ is obtained by doubling a Jordan triple system $J$ iff $L$ is symmetric (i.e. has an involution switching $L_{1}$ and $\left.L_{-1}\right) . V(L)$ is obtained by doubling a Jordan algebra with unit $e$ iff the grading of $L$ is induced by a subalgebra $\mathrm{sl}(2)=\{e, f, h\}(e=e \oplus 0 \oplus 0, f=0 \oplus 0 \oplus e$, $h=0 \oplus \mathrm{Id} \oplus 0$ ).

It is easy to see why Jordan pairs are spontaneously generated by such Lie algebras: from $\left\{\begin{array}{lll}x y & z\end{array}\right\}=[[x y] z]$ in (5.7) we have $V_{x y}=\operatorname{ad}[x y]$, so the triple axiom (5.5) for $V(L)$ becomes

$$
[\operatorname{ad}[x y], \operatorname{ad}[z w]]=\operatorname{ad}[[[x y], z], w]-\operatorname{ad}[z,[[y x], w]]
$$

and therefore falls right out of the Jacobi identity for $L$.

Thus the study of graded Lie algebras leads naturally to Jordan pairs (including triple systems and algebras). From several points of view, Jordan pairs are the most natural Jordan systems; a complete theory has been developed by Ottmar Loos in his book, Jordan pairs [20].

We are saying that if you open up a Lie algebra and look inside, 9 times out of 10 there is a Jordan algebra (or pair) which makes it tick.

VI. Applications to analysis. To appreciate the analytic applications, it is not necessary to be familiar with the concepts involved; it is enough to believe the Halmosian dictum: "important mathematics being spoken here".

The discovery and development of the analytic applications is due primarily to Max Koecher and his students, with important contributions by Kantor, Resnikoff, Rothaus, Vinberg, and others. It has profoundly affected 
the course of Jordan theory (even in its purely algebraic aspects).

REAL ANALYSIS. A geometrically important species of space is a Riemannian symmetric space, which is an analytic Riemannian manifold, each point of which is an (isolated) fixed point of an involutive isometry (the so-called geodesic symmetry about that point). As with any Riemannian manifold $M$, such a space has an affine connection determined by Christoffel symbols $\Gamma_{i j}^{k}$, and at each point $p \in M$ there is an exponential map $\exp _{p}$ of the tangent space $T_{p}(M)$ to the manifold at $p$ down into the manifold itself, such that 0 projects onto $p$ and $x \rightarrow-x$ projects onto the geodesic symmetry.

The self-dual homogeneous cones are an important example of symmetric spaces, and there is a 1-1 correspondence between them and formally real Jordan algebras (real algebras where a sum of squares of elements vanishes only when each of the elements vanishes).

(6.1) EXAMPLE [36], [18]. Let $J$ be a formally real Jordan algebra, and $C(J)$ the positive cone of $J$ (the identity component of the set of invertible elements-equivalently, the set of all exponentials $\left.e^{x}=\Sigma_{0}^{\infty} x^{n} / n !\right)$. Then $C(J)$ is an open subset of $\mathbf{R}^{n}$ and becomes a Riemannian symmetric space. At $p=1$ the geodesic symmetry is simply the inversion $x \rightarrow x^{-1}$, and the exponential map is the ordinary algebraic exponential $\exp _{1}(x)=e^{x}$. Any other point $p$ can be considered as the unit element in its own algebraic system, the isotope $J^{[p]}$, so has geodesic symmetry $x \rightarrow x^{-1[p]}$ and exponential map $\exp _{p}(x)=e^{x[p]}$. The affine connection $\Gamma_{i j}{ }^{k}$ coincides with the structure constants $c_{i j}^{k}$ of the Jordan algebra $J$.

Complex ANAlysis. The complex analogue of a Riemannian symmetric space is a Hermitian symmetric space, a real symmetric space with a complex structure invariant under geodesic symmetry. (Such a space is a complex analytic manifold such that in each tangent space there lives a Hermitian inner product.) These are abstract complex manifolds. In contrast, a bounded symmetric domain is a bounded domain in $\mathbf{C}^{n}$ such that every point is an (isolated) fixed point of an involutive automorphism of the domain. Initially such a domain has no metric, but there is a natural way to introduce one.

6.2 THEOREM [8]. Every bounded symmetric domain naturally carries the structure of a Hermitian symmetric space [of noncompact type] via the Bergman metric derived from the Bergman kernel; all automorphisms of the domain become isometries of this Hermitian structure. Conversely, every Hermitian symmetric space [of noncompact type] is isomorphic to a bounded symmetric domain in $\mathbf{C}^{\mathbf{n}}$.

This reduces the study of certain abstract Hermitian symmetric spaces to the study of more tangible domains in $\mathbf{C}^{n}$. At this point we make a further reduction to an even nicer sort of domain. A bounded homogeneous circled domain is a bounded domain in $\mathbf{C}^{n}$ containing the origin, such that the circling maps $x \rightarrow e^{i t} x$ are automorphisms of the domain for all real $t$, and such that the group of all automorphisms acts transitively on the domain.

These circled domains are actually symmetric domains: at the origin the symmetry is the automorphism $e^{i \pi}=-I d$, and exists at all other points by homogeneity. Conversely, all bounded symmetric domains are isomorphic to circled domains: 
6.3 THEOREM [19], [36], [8]. Every bounded symmetric domain is biholomorphically equivalent to a bounded homogeneous circled domain (its Harish-Chandra realization). A bounded homogeneous circled domain is its own Harish-Chandra realization.

Thus the study of Hermitian symmetric spaces $M$ is reduced to the study of certain round open domains $D$ in $\mathbf{C}^{n}$. We give some examples of such realizations $\rho$.

6.4 EXAMPLE (Upper half plane).

$$
\begin{aligned}
& M=\text { the upper half plane of all } x+i y, y>0(x, y \in \mathbf{R}), \\
& D=\text { unit disc } 1-\bar{z} z>0(z \in \mathbf{C}), \\
& \rho=\text { Cayley transform } z \rightarrow(1+i z) /(1-i z) .
\end{aligned}
$$

6.5 EXAMPLE (Siegel's generalized upper half plane).

$$
\begin{aligned}
M= & \text { all } X+i Y, Y \text { positive definite }\left(X, Y \in M_{n}(\mathbf{R})\right), \\
D= & \text { generalized unit disc of } Z \in M_{n}(\mathbf{C}) \text { with Id }-\bar{Z} Z \\
& \text { positive definite, } \\
\rho= & \text { Cayley transform } Z \rightarrow(\operatorname{Id}+i Z)(\mathrm{Id}-i Z)^{-1} .
\end{aligned}
$$

6.6 EXAMPLES (Koecher's halfspace of a formally real Jordan algebra $J$ ) [18].

$$
\begin{aligned}
M= & H(J)=\text { all } X+i Y, Y \text { in the positive cone } C(J) \\
& (X, Y \in J)(\text { so } H(J)=J+i C(J) \text { is an open subset of } \\
& \text { the complexification } \left.J_{\mathrm{C}} \text { of the Jordan algebra }\right) \\
D= & \text { all elements } z \text { in } J_{\mathrm{C}} \text { for which the operator } \\
& 2 \text { Id }-V(z, z) \text { is positive definite, } \\
\rho= & \text { Cayley transform } Z \rightarrow(1+i Z)(1-i Z)^{-1} .
\end{aligned}
$$

Here the geometry of $H(J)$ is nicely described in Jordan terms: the group Aut $H(J)$ of automorphisms consists of the linear fractional transformations, generated by inversion $z \rightarrow-z^{-1}$, translations $z \rightarrow z+a(a \in J)$, and $z \rightarrow$ $T z$ for those $T$ in the structure group of $J$ which map $C(J)$ onto itself.

Finally, the bounded homogeneous circled domains are categorically equivalent to certain Jordan triple systems. A Hermitian Jordan triple system is a real Jordan triple with complex structure such that the triple product $\left\{\begin{array}{lll}x & y & z\end{array}\right\}$ is $\mathbf{C}$-linear in $x$ and $z$ but $\mathbf{C}$-antilinear in the middle variable $y$, and where the bilinear form $\langle x, y\rangle=\operatorname{trace} V_{x, y}$ is a positive definite Hermitian scalar product.

6.7 THEOREM [19], [36], [21]. There is a natural 1-1 correspondence between bounded homogeneous circled domains and Hermitian Jordan triple systems. If $D$ is a domain with Bergman kernel $K$ the Jordan triple structure is given by 


$$
\begin{aligned}
\langle u, v\rangle & =\left.d_{u} d_{\bar{v}} \log K(z, z)\right|_{z=0}, \\
\{u v w\} & =\Sigma c_{i j k l} u_{i} \bar{v}_{j} w_{k} \vec{e}_{l} \text { for } C_{i j, k, l}=\left.\frac{\partial^{4} \log K(z, z)}{\partial z_{i} \partial \bar{z}_{j} \partial z_{k} \partial \bar{z}_{l}}\right|_{z=0} .
\end{aligned}
$$

Conversely, given a triple system $J$ the domain is obtained as

$$
D=\{x \mid 2 \mathrm{Id}-V(x, x)>0\} .
$$

The geometry and algebra are related by results such as:

The Bergman kernel is $K(z, w)=(1 / \mu(D)) \operatorname{det}^{-1} B(z, w)$.

The Bergman metric at 0 is $\langle u, v\rangle=\operatorname{trace} V_{u, v}$.

The Shilov boundary of $\bar{D}=$ the set of maximal idempotents of $J$.

The affine and holomorphic boundary components of $\bar{D}$ are the faces $e+D \cap V_{0}(e)$ for all idempotents $e$ of $J$.

$D=D_{1} \times \cdots \times D_{r}$ decomposes into irreducible domains iff $J=J_{1} \oplus \cdots \oplus J_{r}$ decomposes into simple triple systems.

The automorphisms of the domain fixing 0 are just the automorphisms of the triple system $\operatorname{Aut}_{0}(D)=\operatorname{Aut}(J)$.

All geometric information about $D$ is encoded in the algebraic structure of the Jordan triple system $J$. This yields a simple direct treatment of such domains which avoids the elaborate machinery of Lie theory (the usual method of analyzing them). For example, the simple Hermitian Jordan triple systems are

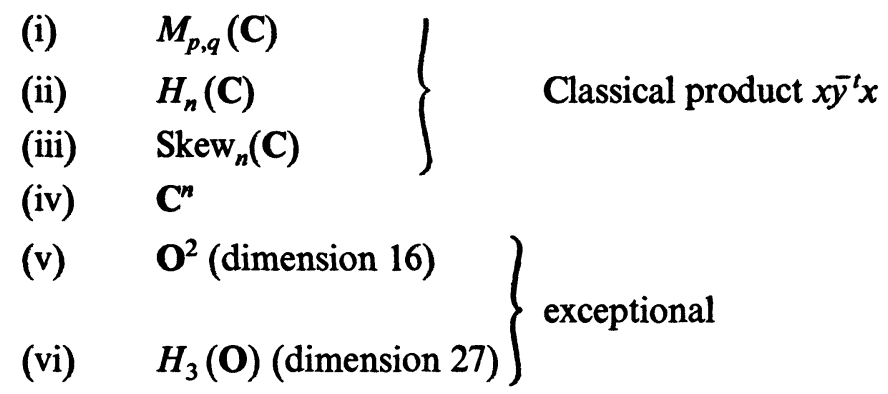

and these correspond to the irreducible domains. The Jordan approach has been generalized by L. Harris [34] and W. Kaup [35] to the infinite-dimensional case. Indeed, Jordan triple systems can answer any question you care to ask about bounded homogeneous circled domains.

VII. Conclusion. Jordan algebras arose as an attempt to capture the algebraic essence of Hermitian matrices (or symmetric elements of an associative algebra with involution). They are close enough to associative algebras to remain tractable, yet they let in just enough generality to include $H_{3}(O)$, thereby yielding connections with exceptional structures in many branches of mathematics. As a result, Jordan methods have proved useful tools in a variety of settings. 


\section{REFERENCES}

Only works referred to in the article are listed here. For more complete bibliographies on linear Jordan algebras see [3] and [10]; for Jordan pairs and triple systems see [20]; for Jordan algebras in projective geometry see [6]; for connections with Lie algebras see [13]; for analytic applications see [8], [18], [19], and [21].

1. A. A. Albert, On a certain algebra of quantum mechanics, Ann. of Math. (2) 35 (1934),

2. E. Alfsen, F. Schultz and E. Störmer, A Gelfand-Neumark theorem for Jordan algebras (to appear).

3. H. Braun and M. Koecher, Jordan Algebren, Springer-Verlag, Berlin, 1966.

4. P. M. Cohn, On homomorphic images of special Jordan algebras, Canad. J. Math. 6 (1954), 253-264.

5. __ Free rings and their relations, Academic Press, New York, 1971.

6. J. Faulkner, Octonion planes defined by quadratic Jordan algebras, Mem. Amer. Math. Soc. No. 104 (1970).

7. F. Gürsey, Algebraic methods and quark structure, Proc. Kyoto Conf. on Math. Prob. in Theoretical Phys. 1975.

8. S. Helgason, Differential geometry and symmetric spaces, Academic Press, New York, 1962.

9. L. K. Hua, On semihomomorphisms of rings and their applications in projective geometry, Uspehi Mat. Nauk 8 (1953), no. 3 (53), 143-148.

10. N. Jacobson, Structure and representations of Jordan algebras, Amer. Math. Soc. Colloq. Publ., vol. 39, Amer. Math. Soc., Providence, R.I.,1969.

11. __ Lectures on quadratic Jordan algebras, Tata Institute Lecture Notes, Bombay, 1969.

12. _ Structure theory for a class of Jordan algebras, Proc. Nat. Acad. Sci. U. S. A. 55 (1966), 243-251.

13. __ Exceptional Lie algebras, Dekker, New York, 1971.

14. P. Jordan, J. von Neumann and E. Wigner, On an algebraic generalization of the quantum mechanical formalism, Ann. of Math. (2) 36 (1934), 29-64.

15. V. Kac, Classification of simple Z-graded Lie superalgebras and simple Jordan superalgebras (to appear).

16. J. Kaplansky, Graded Lie and Jordan algebras (to appear).

17. M. Koecher, On Lie algebras defined by Jordan algebras, Aarhus Univ. Lecture Notes, Aarhus, 1967.

18. __ Jordan algebras and their applications, Univ. Minnesota Lecture Notes, Minneapolis, 1962.

19. , An elementary approach to bounded symmetric domains, Rice Univ. Lecture Notes, Houston, 1969.

20. O. Loos, Jordan pairs, Lecture Notes in Math., vol. 460, Springer-Verlag, New York, 1975.

21. __ Jordan pairs and bounded symmetric domains, Lecture Notes, Univ. of California at Irvine, 1977.

22. K. McCrimmon, Noncommutative Jordan rings, Trans. Amer. Math. Soc. 158 (1971), 1-33.

23. _ A general theory of Jordan rings, Proc. Nat. Acad. Sci. U. S. A. 56 (1966), 1072-1079.

24. __ Jordan algebras with interconnected idempotents, Proc. Amer. Math. Soc. 19 (1968), $1327-1336$.

25. __ Axioms for inversion in Jordan algebras, J. Algebra 47 (1977).

26. On Herstein's theorems relating Jordan and associative algebras, J. Algebra 13 (1969), 382-392.

27. R. Moufang, Alternative körper und der Satz vom Vollständigen Vierseit, Abh. Math. Sem. Univ. Hamburg 9 (1933), 207-222.

28. J. von Neumann, On an algebraic generalization of the quantum mechanical formalism, Mat. Sb. 1 (1936), 415-482.

29. T. A. Springer, Jordan algebras and algebraic groups, Springer-Verlag, New York, 1973.

30 The projective octave plane, Indag. Math. 22 (1960), 74-101.

31. A. Thedy, Right alternative rings, J. Algebra 37 (1975), 1-63. 
32. J. Tits, Algèbres alternatives, algèbres de Jordan, et algèbres de Lie exceptionelles. I, Indag. Math. 28 (1966), 223-237.

33. D. M. Topping, Jordan algebras of selfadjoint operators, Mem. Amer. Math. Soc. No. 53 (1965).

34. L. Harris, Bounded symmetric homogeneous domains in infinite dimensional spaces, Proc. Infinite Dimensional Holomorphy, Lecture Notes in Math., vol. 364, Springer-Verlag, New York, 1974.

35. W. Kaup, Algebraic characterization of symmetric complex Banach manifolds, Math. Ann. (to appear).

36. M. Koecher, Jordan algebras in differential geometry, Proc. Internat. Congress I, Nice, 1970), 279-283.

Department of Mathematics, University of Virginia, Charlottesville, Virginia 22901 